\title{
Measurement of through-going particle momentum by means of multiple scattering with the ICARUS T600 TPC
}

\author{
Journal Article \\ Author(s): \\ ICARUS Collaboration; Ankowski, A.; Badertscher, Andreas; Ge, Y.; Gil Botella, I.; Laffranchi, Marco; Messina, M.; Rubbia, André; \\ et al.
}

Publication date:

2006-11

Permanent link:

https://doi.org/10.3929/ethz-b-000035317

Rights / license:

Creative Commons Attribution 4.0 International

Originally published in:

The European Physical Journal C 48(2), https://doi.org/10.1140/epjc/s10052-006-0051-3 


\title{
Measurement of through-going particle momentum by means of multiple scattering with the ICARUS T600 TPC
}

\author{
The ICARUS Collaboration
}

A. Ankowski ${ }^{1}$, M. Antonello ${ }^{2}$, P. Aprili ${ }^{3}$, F. Arneodo ${ }^{3}$, A. Badertscher ${ }^{4}$, B. Baiboussinov ${ }^{5}$, M. Baldo Ceolin ${ }^{5}$, G. Battistoni ${ }^{6}$, P. Benetti ${ }^{7}$, A. Borio di Tigliole ${ }^{7}$, R. Brunetti ${ }^{7}$ a, A. Bueno ${ }^{8, b}$, E. Calligarich ${ }^{7}$, F. Carbonara ${ }^{9}$, M.C. Carmona ${ }^{8}$, F. Cavanna ${ }^{2}$, P. Cennini ${ }^{10}$, S. Centro ${ }^{5}$, A. Cesana ${ }^{11}$, D.B. Cline ${ }^{12}$, K. Cieślik ${ }^{13}$, A.G. Cocco ${ }^{9}$, C. De Vecchi ${ }^{7}$, A. Dạbrowska ${ }^{3}$, A. Di Cicco ${ }^{9}$, R. Dolfini ${ }^{7}$, A. Ereditato ${ }^{9}$, A. Ferrari ${ }^{10}$, G. Fiorillo ${ }^{9}$,

D. García-Gamez ${ }^{8}$, Y. Ge ${ }^{4}$, D. Gibin ${ }^{5}$, A. Gigli Berzolari ${ }^{7}$, I. Gil-Botella ${ }^{14}$, K. Graczyk ${ }^{1}$, L. Grandi ${ }^{7}$, A. Guglielmi ${ }^{5}$, J. Holeczek ${ }^{15}$, D. Kiełczewska ${ }^{16}$, J. Kisiel ${ }^{15}$, T. Kozłowski ${ }^{17}$, M. Laffranchi ${ }^{4}$, J. Lagoda ${ }^{16}$, B. Lisowski ${ }^{12}$, J. Lozano ${ }^{8}$, M. Markiewicz ${ }^{13}$, A. Martínez de la Ossa ${ }^{8}$, C. Matthey ${ }^{12}$, F. Mauri ${ }^{7}$, A.J. Melgarejo ${ }^{8}$, A. Menegolli ${ }^{7}$, G. Meng ${ }^{5}$, M. Messina ${ }^{4}$, P. Mijakowski ${ }^{17}$, C. Montanari ${ }^{7}$, S. Muraro ${ }^{6}$, S. Navas $^{8}$, J. Nowak ${ }^{1}$, S. Otwinowski ${ }^{12}$, O. Palamara ${ }^{3}$, L. Periale ${ }^{18}$, G. Piano Mortari ${ }^{2}$, A. Piazzoli ${ }^{7}$, P. Picchi ${ }^{18}$, F. Pietropaolo ${ }^{5}$, W. Półchłopek ${ }^{19}$, M. Posiadała ${ }^{16}$, M. Prata ${ }^{7}$, M.C. Prata ${ }^{7}$, P. Przewlocki ${ }^{7}$, A. Rappoldi ${ }^{7}$, G. L Raselli ${ }^{7}$, E. Rondio ${ }^{17}$, M. Rossella ${ }^{7}$, B. Rossi ${ }^{9}$, A. Rubbia ${ }^{4}$, C. Rubbia ${ }^{7}$, P.R. Sala ${ }^{6}$, D. Scannicchio ${ }^{7}$, E. Segreto ${ }^{2}$, Y. Seo ${ }^{12}$, F. Sergiampietri ${ }^{20}$, J. Sobczyk ${ }^{1}$, D. Stefan ${ }^{13}$, J. Stepaniak ${ }^{17}$, R. Sulej ${ }^{21}$, M. Szeptycka ${ }^{17}$, M. Szarska ${ }^{13}$, M. Terrani ${ }^{11}$, F. Varanini ${ }^{5}$, S. Ventura ${ }^{5}$, C. Vignoli ${ }^{7}$, T. Wąchała ${ }^{13}$, H. Wang ${ }^{12}$, X. Yang ${ }^{12}$, A. Zalewska ${ }^{13}$

${ }^{1}$ Institute of Theoretical Physics, Wrocław University, Wrocław, Poland

2 Gruppo collegato INFN and Dipartimento di Fisica, Università dell'Aquila, L'Aquila, Italy

${ }^{3}$ Laboratori Nazionali del Gran Sasso (LNGS) INFN, Assergi, Italy

${ }^{4}$ Institute for Particle Physics, ETH Hönggerberg, Zürich, Switzerland

5 Dipartimento di Fisica, Università di Padova and INFN, Padova, Italy

${ }^{6}$ Dipartimento di Fisica, Università di Milano and INFN, Milano, Italy

7 Dipartimento di Fisica Nucleare e Teorica, Università di Pavia and INFN, via Bassi 6, 27100 Pavia, Italy

8 Departamento de Física Teórica y del Cosmos and Centro Andaluz de Física de Partículas Elementales (CAFPE), Universidad de Granada, Granada, Spain

9 Dipartimento di Scienze Fisiche, Università Federico II di Napoli and INFN, Napoli, Italy

10 CERN, Genève, Switzerland

11 Dipartimento di Ingegneria Nucleare, Politecnico di Milano and INFN, Milano, Italy

12 Department of Physics and Astronomy, University of California, Los Angeles, USA

13 H. Niewodniczański Institute of Nuclear Physics, Kraków, Poland

14 CIEMAT, Departamento de Investigacion Basica, Madrid, Spain

15 Institute of Physics, University of Silesia, Katowice, Poland

16 Institute of Experimental Physics, University of Warszawa, Poland

17 A. Sołtan Institute for Nuclear Studies, Warszawa, Poland

18 Laboratori Nazionali di Frascati (LNF) INFN, Frascati, Italy

19 Department of Electronics, AGH University of Science and Technology, Kraków, Poland

${ }^{20}$ INFN, Pisa, Italy

21 Institute of Radioelectronics, University of Warsaw, Warszawa, Poland

Received: 2 June 2006 / Revised version: 31 August 2006 /

Published online: 24 October 2006 - C S Springer-Verlag / Società Italiana di Fisica 2006 


\begin{abstract}
The ICARUS collaboration has demonstrated, following the operation of a 600 ton (T600) detector at shallow depth, that the technique based on liquid argon time projection chambers is now mature. The study of rare events, not contemplated in the standard model, can greatly benefit from the use of this kind of detectors. In particular, a deeper understanding of atmospheric neutrino properties will be obtained thanks to the unprecedented quality of the data ICARUS provides. However if we concentrate on the T600 performance, most of the $\nu_{\mu}$ charged current sample will be partially contained, due to the reduced dimensions of the detector. In this article, we address the problem of how well we can determine the kinematics of events having partially contained tracks. The analysis of a large sample of atmospheric muons collected during the T600 test run demonstrates that, in case the recorded track is at least one meter long, the muon momentum can be reconstructed by an algorithm that measures the multiple Coulomb scattering along the particle's path. Moreover, we show that momentum resolution can be improved by almost a factor two using an algorithm based on the Kalman filtering technique.
\end{abstract}

PACS. 29.40.Gx; 29.85.+c

\section{Introduction}

Liquid argon time projection chambers (TPCs) are a most promising technique for the study of several fundamental topics of particle physics: neutrino properties, proton decay and dark matter. After an extensive R\&D program, the ICARUS collaboration was able to operate at shallow depth a detector of 600 tons (T600) [1]. The successful completion of a series of technical tests has shown that the liquid argon technique is now mature. The sample of cosmic ray events recorded has provided us with a statistically significant data set of unprecedented quality. Long muon tracks, stopping muons, muon bundles, hadronic and electromagnetic showers as well as low energy events have been studied and results have been published elsewhere [2-7].

The T600 detector is now at the INFN Gran Sasso Laboratory. It is being installed to be operated underground. Its physics program has been reviewed in [8]. Among the different non-accelerator physics topics that can be covered with such a detector, the study of atmospheric neutrinos is particularly important. Thanks to its high granularity, ICARUS can separate, for all neutrino species, charged current and neutral current events down to production thresholds and free of detector biases. Therefore it provides a data set of very high quality (specially in the sub-GeV region) and almost free of systematic uncertainties (which are nowadays the limiting factor in the study of atmospheric neutrino oscillations).

Atmospheric $\nu_{\mu}$ charged current interactions will produce prompt muons with a mean energy of several hundreds of MeV. They will have travel paths of several meters, since minimum ionizing particles deposit about $210 \mathrm{MeV}$ per meter in argon. However, due to the reduced transverse dimensions of the T600 detector, a large fraction of the recorded $\nu_{\mu}$ charged current will be partially contained. Calorimetric measurements will be highly inadequate to study this sort of events. To extract valuable kinematic information from them, we can take advantage of the multiple Coulomb scattering underwent by muons as they propagate through liquid argon. Momentum measurement of partially contained tracks will be performed in a way very similar to the one used in emulsion experiments. To-

\footnotetext{
a Present address: INFN, Torino, Italy

b e-mail: a.bueno@ugr.es
}

gether with this method, we try to apply the technique, known as Kalman filtering [9], in order to obtain a more precise measurement of the particle momenta.

This article is organized as follows: Sect. 2 gives a brief outline of the detector. In Sect. 3 we discuss the technical aspects of the Monte Carlo simulation and event reconstruction. Section 4 describes what we called the classical approach to momentum measurement by means of multiple Coulomb scattering. In Sects. 5 and 6 we briefly introduce the Kalman filter technique and how it can be used to obtain an estimation of momenta for through-going particles. Section 7 compares the performance of the two algorithms. To evaluate the goodness of the Kalman filter method, Sect. 8 describes the results obtained when the Kalman filter algorithm is applied to real data - in our case, a sample of atmospheric stopping muons. Conclusions are finally drawn in Sect. 9.

\section{The 600 ton liquid argon TPC}

The ICARUS T600 liquid argon (LAr) detector [1] consists of a large cryostat split in two identical, adjacent halfmodules, with internal dimensions $3.6 \times 3.9 \times 19.6 \mathrm{~m}^{3}$ for each of them. Each half-module is an independent unit housing an internal detector composed by two time projection chambers, a field shaping system, monitors, probes, and two arrays of photo-multipliers. Externally the cryostat is surrounded by a set of thermal insulation layers. The TPC wire read-out electronics is located on the top side of the cryostat. The detector layout is completed by a cryogenic plant made of a liquid nitrogen cooling circuit to maintain uniform the LAr temperature and of a system of LAr purifiers.

A liquid argon TPC detects the ionization charge released at the passage of charged particles in the volume of LAr, thus providing three-dimensional image reconstruction and calorimetric measurement of ionizing events. The detector, equipped with an electronic read-out system, works as an "electronic bubble chamber" employing LAr as ionization medium.

An uniform electric field applied to the medium makes the ionization electrons drift onto the anode; thanks to the low transverse diffusion of the ionization charge, the elec- 
tron images of ionizing tracks are preserved. Successive anode wire planes, biased at a different potential and oriented at different angles, make possible the three-dimensional reconstruction of the track image.

In each T600 half-module, the two identical TPCs are separated by a common cathode. Each TPC consists of three parallel wire planes: the first, facing the drift region, with horizontal wires (induction plane); the other two with the wires at $\pm 60^{\circ}$ from the horizontal direction (induction and collection planes, respectively). The wire pitch is $3 \mathrm{~mm}$. The maximum drift path (distance between the cathode and the wire planes) is $1.5 \mathrm{~m}$ and the nominal drift field $500 \mathrm{~V} / \mathrm{cm}$.

Each wire of the chamber is independently digitized every $400 \mathrm{~ns}$. The electronics was designed to allow for continuous read-out, digitization and independent waveform recording of signals from each wire of the TPC. Measurement of the time when the ionizing event occurred (so called " $t_{0}$ time" of the event), together with the electron drift velocity information, provides the absolute position of the tracks along the drift coordinate. The $t_{0}$ can be determined by detection of the prompt scintillation light produced by ionizing particles in LAr [10].

\section{Monte Carlo simulation and event reconstruction}

Our goal is to understand how accurately we can measure the momentum of charged particles that traverse the T600 detector without stopping. To this purpose, we have carried out a full simulation of muons that span a momentum range going from $250 \mathrm{MeV}$ up to $6 \mathrm{GeV}$. This is the relevant energy range for the study of atmospheric neutrinos. Muons have been generated at the detector center assuming an isotropic distribution in angle.

The FLUKA package [11] has been used to simulate all relevant physics processes (multiple Coulomb scattering, delta ray production, muon decay, energy loss by ionization, ...). The detector geometry has been simulated according to the detector description given in Sect. 2. Special care was put in reproducing the same experimental conditions met when the T600 test at shallow depth was carried out. Therefore we included a detailed simulation of the noise and of the electronic response of the detector.

The event reconstruction proceeded as follows: The wire output signals were used to identify hits (segment of track whose energy is recorded by a wire). Hits are fit in order to extract physical information (deposited charged, time and position). In a latter step, hits are grouped into clusters. From them, we reconstruct two-dimensional tracks for each of the three wire planes (one collection and two induction views). Finally the spatial coordinates of the charged track (3D reconstruction) are obtained matching the hits of the $2 \mathrm{D}$ tracks previously reconstructed. The key point for $3 \mathrm{D}$ reconstruction is the fact that the drift coordinate is common to all three planes. This redundancy allows one to associate hits from different planes to a common energy deposition. 3D tracks are used to extract the information provided by multiple Coulomb scattering. In particular, the use of 3D tracks guarantee that all segments are of equal length. To get a measurement of the track momentum, it is equivalent to use either the angles in space or the projected ones [12]. However, given that we have a better precision on the determination of the drift time coordinate, we observed that, to obtain a very good resolution in our measurement, it is enough to use the projected multiple scattering angle in the plane defined by the drift direction and, for example, the collection wires. The resolution on the momentum measurement depends on the number of reconstructed segments, in other words, on the angle between the track direction and the projection plane. The maximum angle that will allow one to measure the momentum with a reasonable resolution is clearly a detector dependent issue. Given the fine granularity (3 mm pitch) of the T600 detector, tracks with angles with respect to the projection plane as large as $\sim 70$ degrees still provide enough hits to get a precise measurement of the momentum.

The same procedure was used to reconstruct the real data sample of stopping muons used in Sect. 8. To exemplify how the reconstruction software works, Fig. 1 shows a view of a $0.5 \mathrm{GeV}$ stopping muon (left panel) and its three-dimensional reconstruction (right panel).
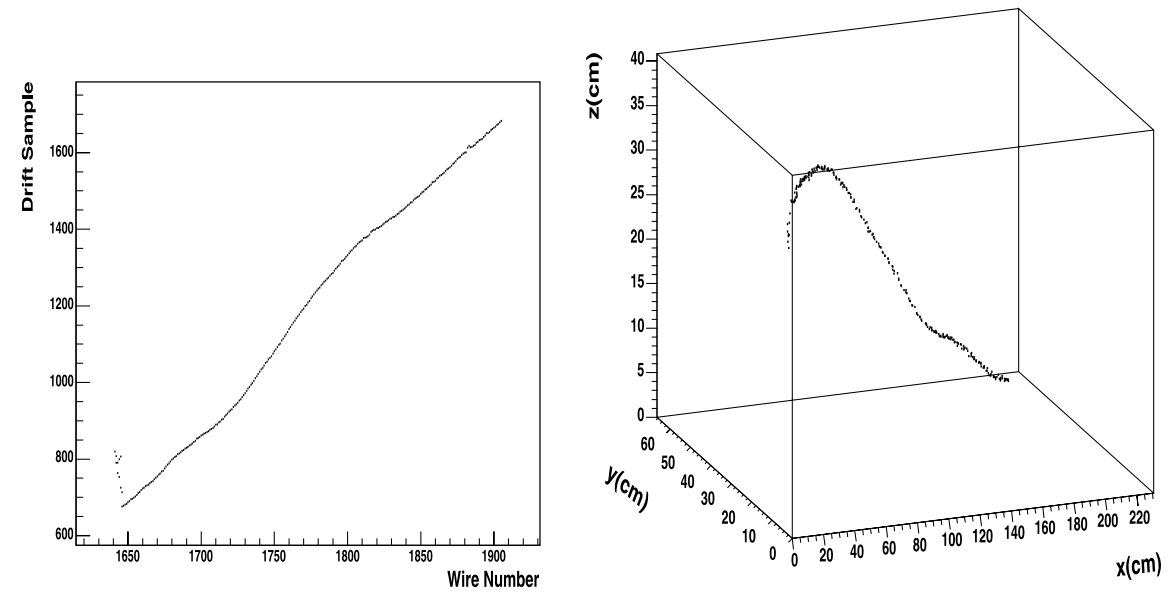

Fig. 1. Collection (2D) view of a typical stopping muon. 3D reconstruction of the same muon event (right) 


\section{The classical approach to momentum measurement}

We discuss what hereafter will be referred to as the classical approach to momentum measurement. It profits from the fact that a charged particle traversing a medium is deflected through many small angle scatterings, $\theta_{i}$. To estimate the momentum of a charged particle we use the following analytical expression [12]:

$$
\theta_{0}^{\mathrm{rms}}=\frac{13.6 \mathrm{MeV}}{\beta c p} z \sqrt{\frac{l}{X_{0}}}\left[1+0.038 \ln \left(\frac{l}{X_{0}}\right)\right]
$$

where $\theta_{0}^{\text {rms }}$ is the width of the Gaussian approximation used for the central $98 \%$ of the projected angular distribution. $p, \beta$ and $z$ are the momentum, velocity and charge of the incident particle. $X_{0}$ is the radiation length and $l$ the considered segment length.

The procedure used to measure the momentum of the particle is as follows.

1. The whole track is split into segments of a fixed length.

2. For each segment, hits belonging to $\delta$-rays are searched for and tagged, such that they can be excluded from the analysis. Otherwise delta ray hits can sensibly distort the determination of the segment direction.

3. The remaining hits in each segment are fitted to a straight line, providing the segment direction.

4. For each consecutive pair of segments, the scattering angle is calculated as the difference between their angles.

5. We compute the RMS of the scattering angle distribution, after cutting out $2 \%$ of the tails (since only the $98 \%$ central interval of the Molière distribution is Gaussian).

6. Finally to calculate the value of $\theta^{\mathrm{rms}}$, we consider all the angles but those whose distance to the mean is larger than 2.5 times the RMS value.

The measured RMS of the scattering angles distribution, $\theta_{\text {meas }}^{\text {rms }}$, is related to the "pure" Coulomb scattering $\theta_{0}^{\text {rms }}$ by the following expression:

$$
\left(\theta_{\text {meas }}^{\text {rms }}\right)^{2}=\left(\theta_{0}^{\text {rms }}\right)^{2}+\left(\theta_{\text {noise }}^{\text {rms }}\right)^{2},
$$

where $\theta_{\text {noise }}^{\text {rms }}$ is the angular detector resolution on the difference of two measured segment angles. In our case, it corresponds to the spatial resolution in the drift coordinate, $\sigma$, which is related to the error on the determination of each individual hit time. This magnitude was measured during the T600 run, using cosmic ray muons and test pulse data, and found to be about $400 \mu \mathrm{m}$. The noise contribution does not depend on the track momentum. It only depends on the segment length $\left(\theta_{\text {noise }}^{\text {rms }} \propto l_{\text {seg }}^{-3 / 2}\right)$. Substituting in (2), we get

$$
\begin{aligned}
\theta_{\text {meas }}^{\text {rms }}= & \sqrt{\left(\theta_{0}^{\text {rms }}\right)^{2}+\left(\theta_{\text {noise }}^{\text {rms }}\right)^{2}} \\
= & \left\{\left(\frac{13.6 \mathrm{MeV}}{\beta c p} z \sqrt{\frac{l}{X_{0}}}\left[1+0.038 \ln \left(\frac{l}{X_{0}}\right)\right]\right)^{2}\right. \\
& \left.+\left(C \cdot l^{-3 / 2}\right)^{2}\right\}^{1 / 2},
\end{aligned}
$$

where $C$ is just a proportionality constant for the noise.
To extract the track momentum, we measure $\theta_{\text {meas }}^{\text {rms }}$ for different segment lengths $(l)$. A fit to those values, using (3), provides an estimation of $p$ and $C$, which are taken as free parameters. This procedure allows one to compute the momentum for each single track, since no other assumptions are made. In addition, with this original approach, we avoid the usual problem of choosing an optimal segment length for the determination of the momentum.

As an example, Fig. 2 shows the result obtained when this procedure is applied to a simulated $3 \mathrm{GeV}$ muon. The triangles correspond to the experimentally measured RMS of the scattering angles for different values of the segment length. The curve indicates the fit result. The rising up at low values of $l$ indicates the region where the contribution from detector resolution dominates, whereas at high values of $l$ the main contribution comes from pure Coulomb scattering.

In this new approach, the key point to compute the momentum on a track by track basis is to decide the set of segment lengths that will be included in the fit. The minimum segment length should be such that effects due to multiple scattering emerge from detector noise. The optimal value for this minimum segment length is $5 \mathrm{~cm}$. The maximum segment length should be short enough to allow for as much entries as possible inside the angle distribution used to compute $\theta_{\text {meas }}^{\text {rms }}$. This last value clearly depends on the recorded track length. To improve our results, we decided to split our sample into tracks having lengths longer than 2.5 meters and tracks shorter than 2.5 meters. In fact, this corresponds to a muon momentum cut at around $600 \mathrm{MeV}$. For long tracks we have used 13 segment lengths inside the interval $[5 \mathrm{~cm}, 35 \mathrm{~cm}]$. For those tracks longer than 5 meters we have used only this length, as using the whole track does not improve the results. For shorter tracks we used 10 segment lengths inside the interval $[5 \mathrm{~cm}, 25 \mathrm{~cm}]$.

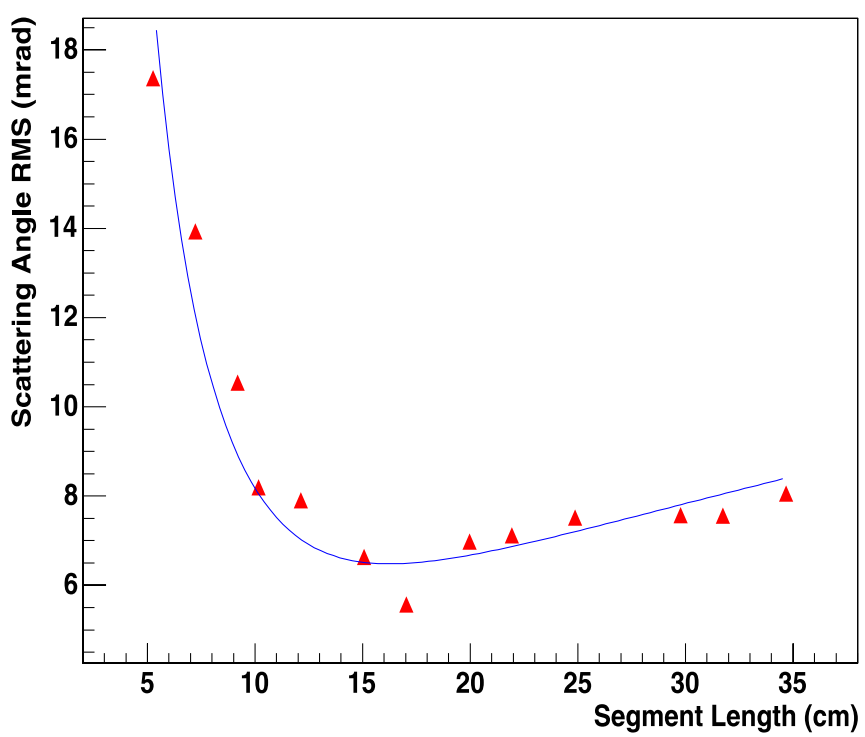

Fig. 2. Fit of the $\theta_{\mathrm{rms}}^{\text {meas }}$ as a function of the segment length for a single $3 \mathrm{GeV}$ muon track 

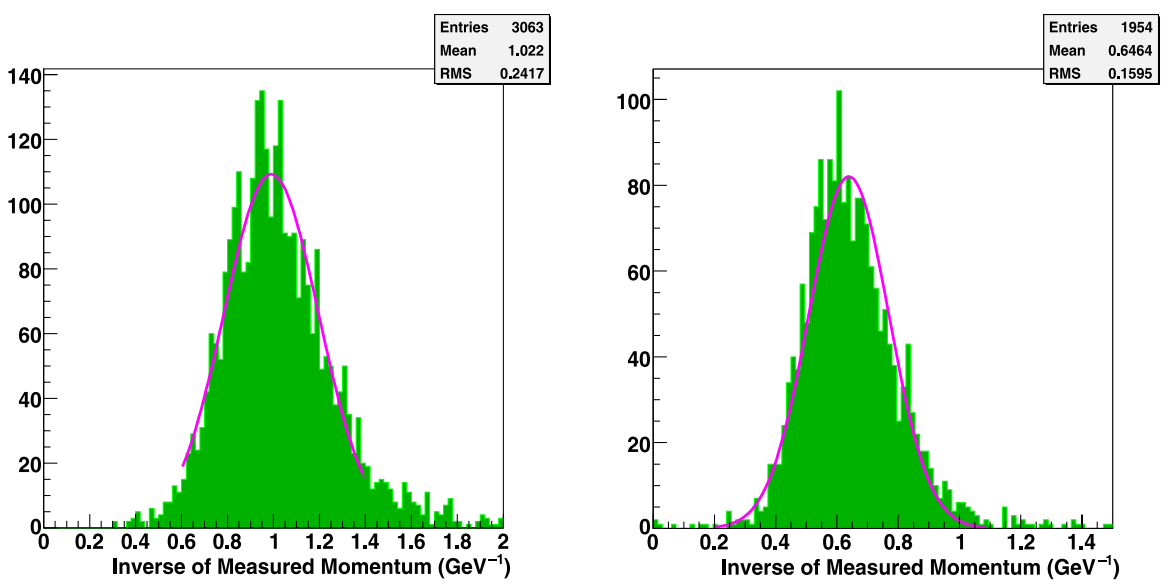

Fig. 3. Histograms of the inverse of the measured momentum for 1.5 and $2 \mathrm{GeV}$ simulated muons

Assuming that the distribution of RMS of scattering angles is Gaussian, it can be demonstrated that the momentum is distributed according to the following function:

$$
\frac{1}{a_{0} p^{2}} \exp \left(\frac{\frac{1}{p}-\frac{1}{a_{1}}}{a_{2}}\right)^{2},
$$

where $a_{1}$ gives an estimate of the momentum average.

Moreover, the momentum resolution $\Delta p$ is estimated from the RMS of the distribution obtained after computing the different scattering angle RMS, given that

$$
\frac{\Delta \theta^{\mathrm{rms}}}{\theta^{\mathrm{rms}}} \equiv \frac{\Delta p}{p} .
$$

Figure 3 shows the distributions of $\frac{1}{p}$ together with the Gaussian fit, for two of the considered momenta.

The classical multiple scattering method, as described so far, obviously underestimates the particle momentum (see discussion in Sect. 7). This is due to the fact that as the particle propagates in liquid argon, it loses energy by means of ionization and therefore its momentum decreases. Since this method does not include any kind of compensation, the final result corresponds to an average reduced momentum and not to the momentum the particle had when it first entered the detector. The latter can be estimated performing an a posteriori correction since we know the track length and the mean energy deposition of a charged particle in liquid argon. In the next section, we introduce a different approach, able to take into account energy losses along the muon track.

\section{The Kalman filter}

The Kalman filter [9] was originally proposed as an algorithm that deduces an optimum estimate of the past, present or future state of a dynamic system. It uses a time sequence of measurements of the system behavior, information about its initial condition plus a statistical model that characterizes the system and the measurement errors.
It provides a tool to separate random noises from signals in statistical processes.

This algorithm has been used extensively in particle physics since it was first proposed as an alternative solution for track and vertex fitting and to estimate momenta [13]. Typically this technique has been used in association with data provided by magnetized detectors. We now explore the possibility to apply the Kalman filter algorithm in an homogeneous, non-magnetized environment (the one offered by the T600) in order to get a measurement of the momentum of charged particles. The particle momentum is extracted from the information provided by the distribution of angles arising from multiple Coulomb scattering process. The Kalman filter helps to disentangle the noise introduced by the detector resolution. In parallel, it takes into account the energy loss along the track, thus improving the determination of its momentum.

The power of the method lies on the fact that all previous measurements are taken into account to predict the future dynamical behavior of the system. Therefore, unlike the case of what we called the classical approach, where the set of reconstructed track segments is just a collection of unrelated measurements, the Kalman filter does take correlations into account (in particular, energy losses are automatically included), and therefore we expect, a priori, an improved estimate of both the momentum and its resolution.

\subsection{The Kalman filter parameters}

The application of the Kalman filter technique requires the definition of a certain set of vectors and matrices (see [13] for a detailed description of the algorithm concept and the relevant equations). The chosen values throughout our calculation are as follows.

1. State vector. To make the analysis, the track is split in segments of a certain length, and in each intersection we consider several magnitudes that will conform our state vector. In each of those segments the hits are fitted to a straight line. The parameters of the fit will define the state vector.

In the following, we will consider a three coordinate system, with our particle traveling in the $z$ direction, 
and being $x$ and $y$ perpendicular to this direction. The state vector will be made of five variables.

- The inverse of the momentum of the particle. This magnitude is related with the angle between the current segment and the next one through the multiple scattering formula (see (1)).

- The position of the particle in the plane (two components: $x, y$ ).

- The slope of the track in the plane (two components: $\left.\frac{\mathrm{d} x}{\mathrm{~d} z}, \frac{\mathrm{d} y}{\mathrm{~d} z}\right)$.

Hence,

$$
\mathbf{x}_{k}=\left(\begin{array}{c}
\frac{1}{p} \\
x \\
y \\
\frac{\mathrm{d} x}{\mathrm{~d} z} \\
\frac{\mathrm{d} y}{\mathrm{~d} z}
\end{array}\right) .
$$

2. Transportation matrix. The propagation matrix performs a straight line extrapolation and does not change the slopes, that is, assumes no interaction. The inverse of the momentum is propagated by subtracting from the momentum the energy lost along the segment length. This energy loss can be directly computed from the charge deposited in the wires by the particle along its path. We have

$$
\mathbf{F}_{k}=\left(\begin{array}{ccccc}
\frac{1}{1-\frac{E_{\text {lost }}}{p}} & 0 & 0 & 0 & 0 \\
0 & 1 & 0 & \Delta z & 0 \\
0 & 0 & 1 & 0 & \Delta z \\
0 & 0 & 0 & 1 & 0 \\
0 & 0 & 0 & 0 & 1
\end{array}\right) .
$$

3. Measurement vector and matrix. The measurement vector is similar to the state vector. The only difference is in the first row, as momentum cannot be measured directly. The measured magnitude is instead the angle difference between the incoming and outgoing trajectories, that is related with the momentum through (1). This difference is taken into account in the measurement matrix, and hence, measurement vector and measurement matrix are given by

$$
\mathbf{m}_{k}=\left(\begin{array}{c}
\theta_{0} \\
x \\
y \\
\frac{\mathrm{d} x}{\mathrm{~d} z} \\
\frac{\mathrm{d} y}{\mathrm{~d} z}
\end{array}\right), \quad \mathbf{H}_{k}=\left(\begin{array}{ccc}
C & & \\
& 1 & \\
& 1 & \\
& & 1 \\
& & 1
\end{array}\right) .
$$

Here $C$ is the multiple scattering constant that multiplies $\frac{1}{p}$ in (1).

4. Covariance matrices. The last ingredient for the method is the choice of the covariance matrices. The covariance matrix for the system noise has been taken from [14] where the authors derive, in a simple and intuitive way, the track parameter covariance matrix due to multiple scattering. They obtain all the matrix elements for two experimentally relevant track parameteri- zations, the first of them being the one we are using: $x$ and $y$ slopes and intercepts.

In the covariance matrix for the detector noise, we have considered no correlations between the components of the state vector, and hence the matrix is diagonal. The value for each point is just the measurement error. This amounts to $400 \mu \mathrm{m}$.

\section{Test of the Kalman filter method on a Monte Carlo sample}

To assess the correctness of the choices made in the previous section in order to build a Kalman filter algorithm suitable to be applied to an homogeneous non-magnetized detector, we have simulated sets of 1000 Monte Carlo muons for each of the following momenta: $0.25,0.5,1.0,1.5,2.0$ and $3.0 \mathrm{GeV}$.

As explained in Sect. 5, an initial state must be supplied for the method to work. It can be thought, a priori, that the input guess for the initial momentum is an important choice that may influence the final value of the momentum measurement. In practice this is avoided by giving a large enough error to the momentum in the first plane, such that its value becomes irrelevant. This has been tested by carrying out a Kalman filter with a random initial state as input. The obtained momentum, close to the real one, was used as input for a second Kalman filter. The final momentum does not differ significantly from the value used as input.

The only important choice for the method is the segment length to be used in the analysis. This must be as short as possible in order to guarantee a large enough number of points, but at the same time segments must be long enough in order to allow multiple scattering effects to emerge over the detector noise. Hence a compromise must be reached.

A priori, the most ambitious idea would be to consider just single hits, as in this way all the information is taken into account and no average is done. But when the segment length is too short, the noise in the measurements is too high, hiding multiple scattering effects. Therefore the momentum cannot be properly estimated. We have seen that to optimize our results it is advisable to use three different segment lengths for each track. For all tracks, we have considered segment lengths of $10,12.5$ and $15 \mathrm{~cm}$. The final momentum value is obtained by averaging the momentum computed for each of the selected segment lengths.

Figure 4 shows the calculated momentum distribution for two Monte Carlo momenta. Each histogram entry corresponds to a reconstructed muon track. A Gaussian has been over-imposed to each histogram. The Gaussian curve fits very well the outline of the histogram. This clearly proves that the Kalman filter technique allows one to have a precise measurement of the muon momentum on a track by track basis.

We note here an intrinsic limitation of the Kalman filter method. It is well known that deviations from the original trajectory, due to multiple scattering, become smaller as momentum increases. Detector noise starts playing a more 

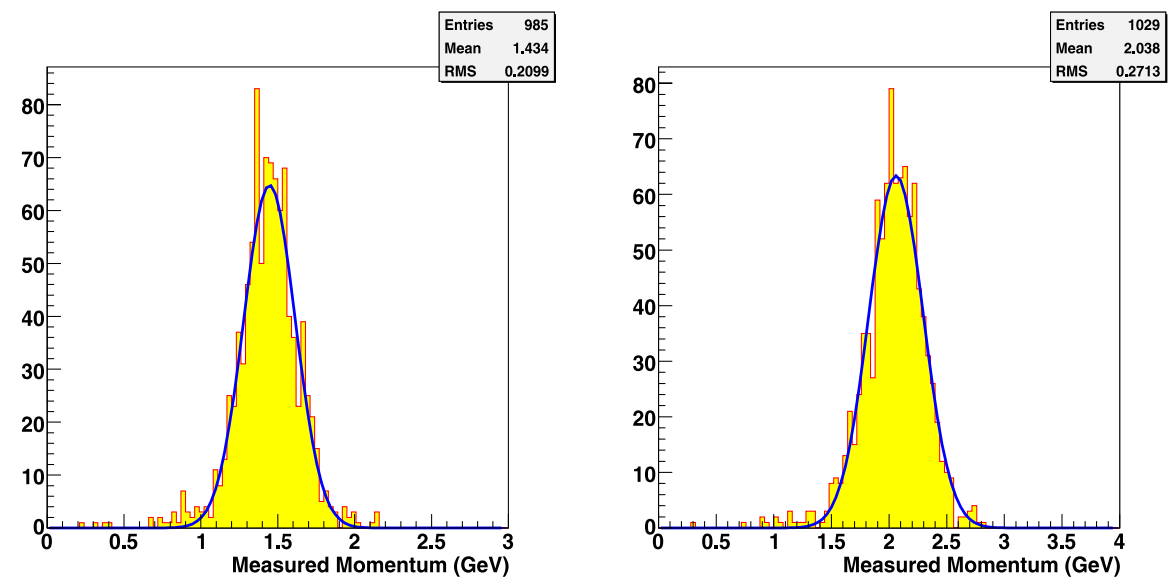

Fig. 4. Momentum distributions as given by the Kalman filter for simulated muons of 1.5 and $2.0 \mathrm{GeV}$

important role and it becomes necessary to go to longer segment lengths to observe multiple scattering effects. Therefore for much higher momenta, a different set of segment lengths should be used. In the limit of very high momentum, $O(10 \mathrm{GeV})$, when multiple scattering effects are small, the Kalman filter procedure is not able to give a proper estimation of the particle momentum.

\section{Comparison of the performance of the classical and Kalman filter approaches on a Monte Carlo sample}

We have discussed separately two algorithms aiming at measuring the momentum of partially contained tracks. To summarize the most relevant points, we now compare the

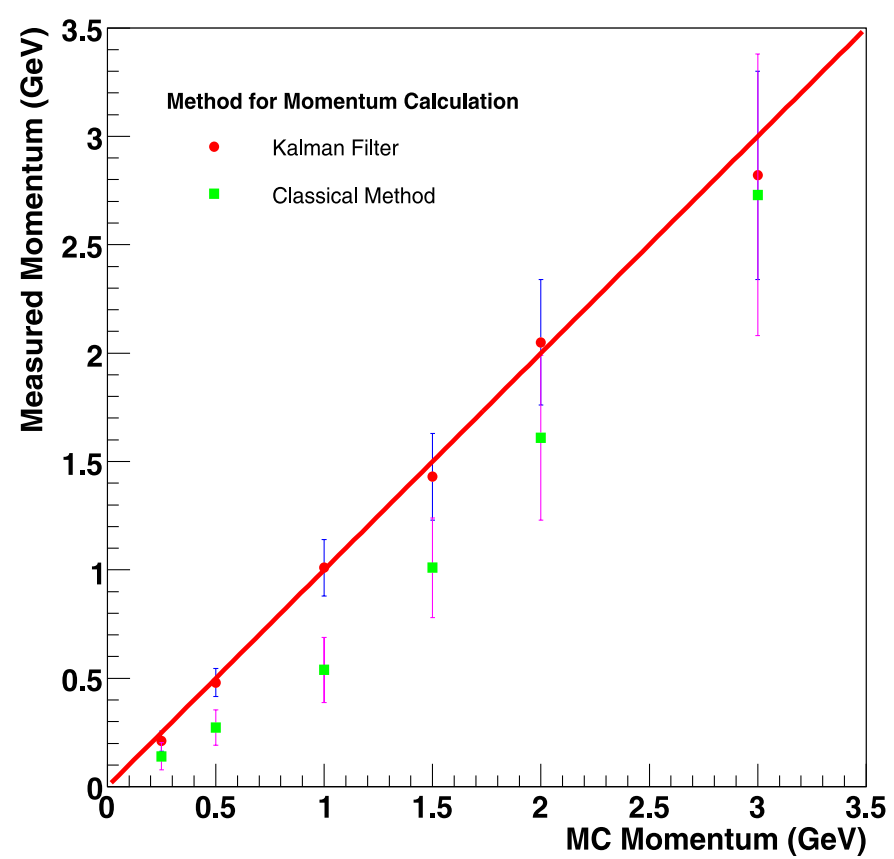

Fig. 5. Comparison between the calibration curves for classical and Kalman filter methods performance of both methods. The comparison is based on Monte Carlo data. Figures 5 and 6 show the calibration curve and the resolutions obtained using the two methods.

In Fig. 5, the solid line shows the case where $P_{\text {measured }}$ $=P_{\text {Monte Carlo }}$. The points from the classical analysis are clearly below the line because of the energy lost by the muon along its path. For this approach, the error bars correspond to the RMS of each single distribution (similar to those shown in Fig. 3).

The Kalman filter gives a much better estimation, since it takes into account energy losses. For this algorithm, the error is taken once again as the RMS of the individual momentum distributions similar to those of Fig. 4. Within one standard deviation, the Kalman filter does not underestimate muon momentum. Therefore, contrary to the classical method where we calculate the mean momentum

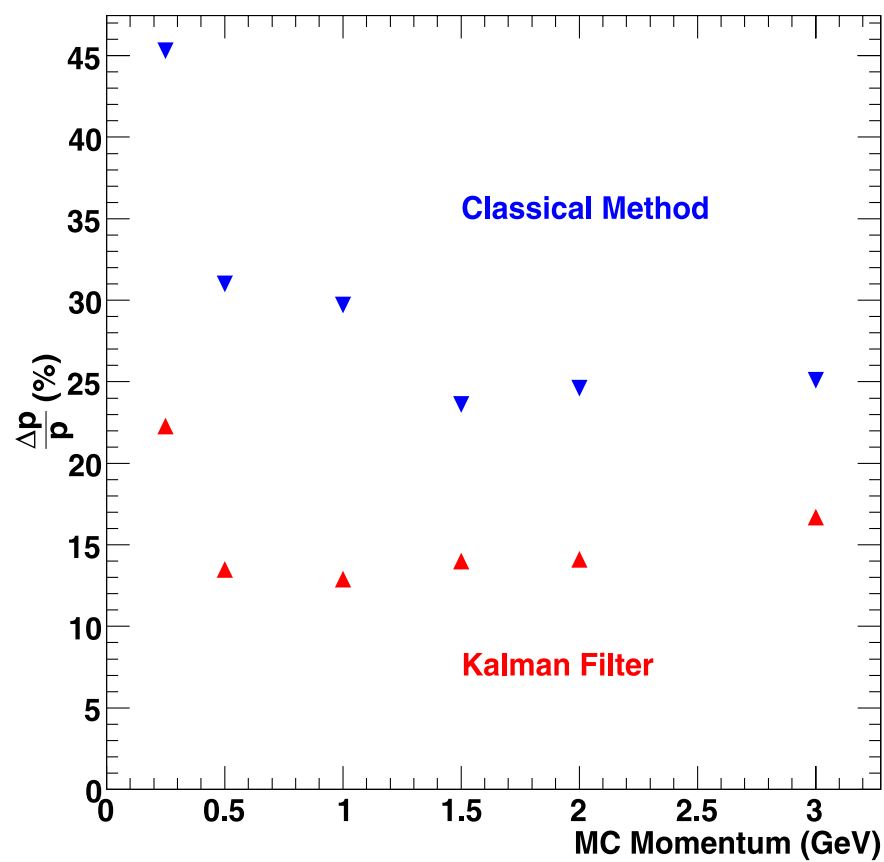

Fig. 6. Comparison between the resolutions for classical and Kalman filter methods 
along the track, with the Kalman filter the momentum at each plane can be calculated, and in particular, we can get a measurement of the most relevant magnitude, namely the momentum at the initial plane.

Figure 6 shows the momentum resolution. For the classical approach it is on the range $25 \%-20 \%$. There is an increase of the resolution at the edges: for small momenta, where the range of lengths and the number of segments is small, and for high momenta, where the effect of the multiple scattering decreases.

With the Kalman filter technique, the resolutions are around $15 \%$ for the whole range of studied momenta, except at $250 \mathrm{MeV}$ where the resolution is close to $25 \%$. This increase is easily understandable given the short track lengths involved. A $250 \mathrm{MeV}$ muon has a typical length of less than 1 meter. The amount of planes in which it can be split is small. Hence the method does not have enough information to provide an accurate measurement. In spite of that, $250 \mathrm{MeV}$ events are typically fully contained and measured by calorimetry with a very good resolution. The inclusion of this energy in the analysis was motivated by the aim to find the limits of the method.

We have also studied how the momentum resolution gets affected as the recorded track length gets shorter. This is specially important for the case of several $\mathrm{GeV}$ muons and for short tracks very close to the detector edges. Figure 7 shows, for the range of considered momenta, the expected resolutions when recorded tracks are 50, 100, 150, 200 and $250 \mathrm{~cm}$ long. We observed that, for all lengths, the momentum scale is not underestimated on average. Resolutions keep themselves in the interval $20 \%-25 \%$. Only for the shortest considered tracks $(50 \mathrm{~cm})$, the resolution worsens up to $30 \%$. We conclude that, for tracks of a few $\mathrm{GeV}$, the Kalman filter allows one to have a reasonable estimate

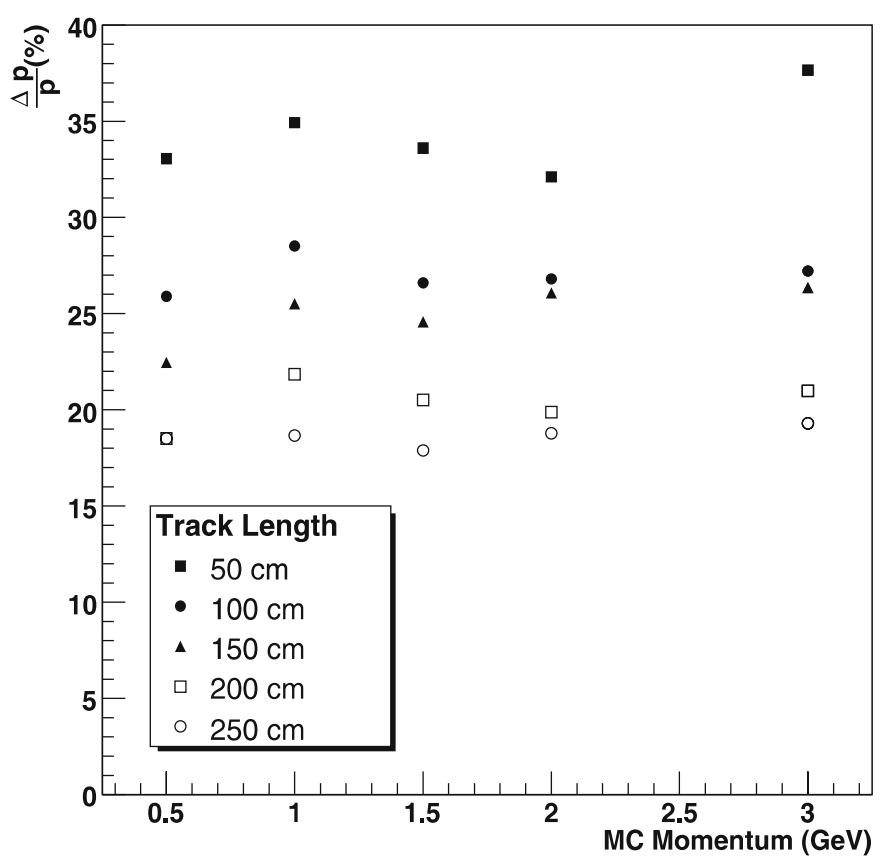

Fig. 7. Kalman filter algorithm: Monte Carlo momentum resolution as a function of the recorded track length of the charged particle momenta even if the recorded tracks are no longer than a meter. This is a crucial result, in particular for detectors of reduced sized as the T600, since this will allow one to enlarge the size of the fiducial volume. The application of this technique sensibly increases the statistical sample of upward- and downward-going atmospheric neutrinos useful for physics analysis (on average, muons emerging from a charged current interaction will propagate through about 2 meters of argon; therefore knowing that a m.i.p. deposits $200 \mathrm{MeV}$ per meter, it is clear that muons above $400 \mathrm{MeV}$ will be mostly non-contained).

In conclusion, with the classical approach we expect resolutions in the range $25 \%-20 \%$ and a underestimation of the momentum value. The results obtained with the Kalman filter do not underestimate momentum and improve the resolution by almost a factor two. In addition, we notice that both methods exhibit a similar behavior at low energies, with an increase in the error with respect to the almost constant one for the rest of energies. This behavior appears at $500 \mathrm{MeV}$ for the classical method and only at $250 \mathrm{MeV}$ for the Kalman filter. In both cases the error increase has to do with the length of the tracks and the small amount of available segments.

\section{Kalman filter application to a set of stopping atmospheric muons}

So far, we have just relied on Monte Carlo data to show that the Kalman filter is an optimal tool to estimate the momentum of partially contained tracks. We now try to assess the goodness of this algorithm by applying it to real data. The sample we take as reference corresponds to a set of stopping atmospheric muons collected on the summer of 2001 during a T600 technical run on surface. The initial set contains the 2690 events used in [5]. We impose the condition that, in the collection view, muons should have at least 60 hits and a minimum track length of $50 \mathrm{~cm}$. These requirements leave us with a final sample of 1009 muons.

Given the characteristics of the selected muons, the range of considered momenta spans from about $200 \mathrm{MeV}$ up to $800 \mathrm{MeV}$ (being the mean value $400 \mathrm{MeV}$ ). For higher momenta, the sample of recorded muons is not statistically significant. The Monte Carlo tells us that the Kalman filter performance for the lower momentum range is not as good as the one expected for muons above $1 \mathrm{GeV}$. However, a demonstration that the method performs reasonably well in the low momentum range will give us confidence that the algorithm can also be applied at higher momenta.

The selected data have been reconstructed following the procedure outlined in Sect. 3. Being fully contained, the momentum of those muons can be very accurately measured using a calorimetric approach. When computing momentum by means of calorimetry, recombination effects should be taken into account. Equation 8 relates measured and deposited charges, $R$ being the electron recombination factor in liquid argon:

$$
Q_{\text {meas }}=R \cdot Q_{\text {dep }} \cdot \mathrm{e}^{\frac{t-t_{0}}{\tau}} .
$$




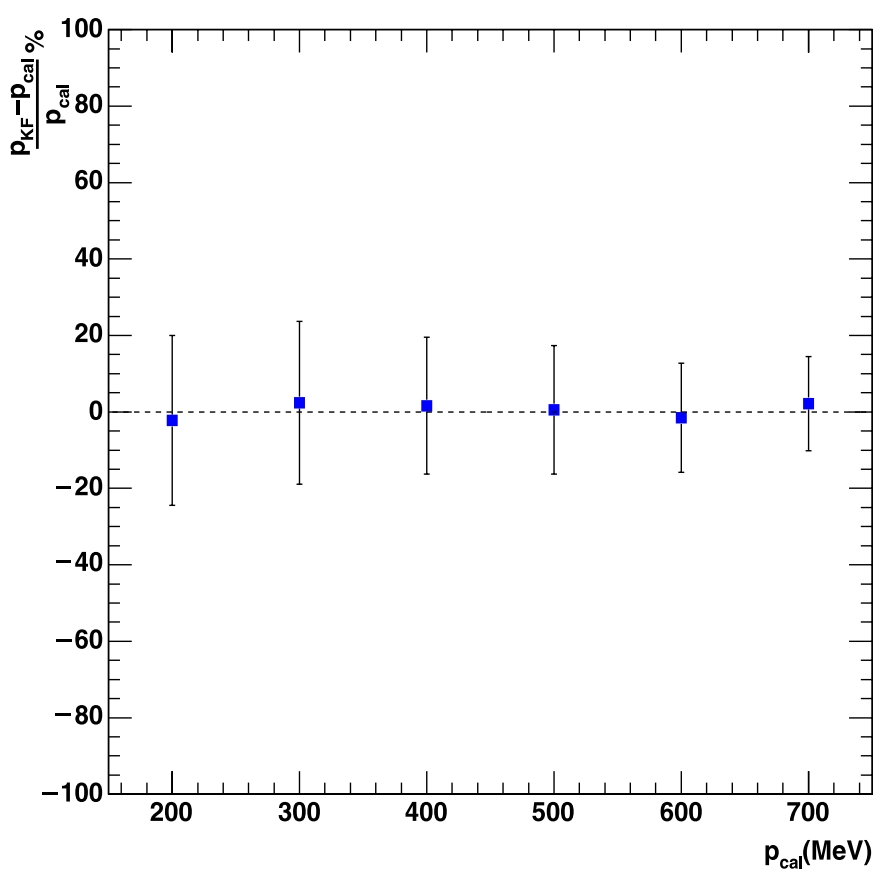

Fig. 8. Dispersion of the Kalman filter measurements with respect to the momentum measured by calorimetry, $p_{\text {cal }}$. The filled squares represent the mean value of the dispersion distribution. The error bars correspond to the RMS

We take from [2] the value $R=0.71 . \tau$ stands for the drift mean life of the electrons and its value is taken from [3]. The error associated to $\tau$ is propagated to the momentum measured by calorimetry. It amounts to less than five per cent.

In our approach we consider the momentum measured by calorimetry, $p_{\text {cal }}$, as the true momentum value. On a track by track basis, we compare the momentum obtained with the Kalman filter, $p_{\mathrm{kf}}$, to $p_{\text {cal }}$. The results are plotted in Fig. 8. It shows the dispersion of the Kalman filter measurements with respect to $p_{\text {cal }}$. For each of the six momentum bins into which we group the data, the mean dispersion is very close to zero. The error bars correspond to the RMS of each individual distribution. For the lowest momenta, the error is around $20 \%$. As the momentum increases, the error bar diminishes, reaching a minimum value of about $12 \%$. These results are in very good agreement with the resolutions obtained, for the low momentum range, in our Monte Carlo simulation (see Fig. 6). In view of these results, we conclude that real data confirm the fact that the Kalman filter algorithm, when combined with multiple Coulomb scattering information, is an optimal method to obtain an accurate measurement of partially contained track momenta.

\section{Conclusions}

We have addressed the problem of measuring the momentum of partially contained charged particles recorded with the 600 ton ICARUS detector. This is a fundamental issue in our effort to study very precisely atmospheric neutrinos.
We have analyzed the performance of two independent approaches.

- The one referred to as classical extracts the momentum from the distribution of scattering angles measured along the particle trajectory. We have proposed an algorithm that avoids defining an optimized segment length for the computation of the scattering angles.

- The second method is based on a Kalman filter. We have explored, for the first time, the possibility to apply this algorithm in an homogeneous, non-magnetized environment. This method takes all measurements into account to predict the future dynamical behavior of the considered system. Therefore, unlike the classical approach, where the set of reconstructed track segments is just a collection of unrelated measurements, the Kalman filter does take correlations into account (in particular, energy losses are automatically included).

With the classical method, the momentum is underestimated (since energy losses are not considered). The expected resolutions lie around $25 \%$ for a range of momenta ranging from $250 \mathrm{MeV}$ up to $3 \mathrm{GeV}$. With the Kalman filter, momentum is not underestimated and the expected resolutions improve by almost a factor two those obtained with the classical method. We have also observed that, for tracks with momentum in the vicinity of $1 \mathrm{GeV}$ or higher, the Kalman filter provides a reasonable momentum measurement, even in the case the recorded tracks are around one meter long.

To assess the goodness of the Kalman filter approach, we have used a set of real data composed of atmospheric muons that stop inside the detector. The momentum of those muons is very precisely measured by calorimetry. The momentum estimation provided by the Kalman filter shows a very good agreement with calorimetric measurements, proving the validity of the method. We therefore conclude that the Kalman filter approach is an excellent tool to get precise kinematics information of the set of partially contained atmospheric neutrino events that we expect to record at the Gran Sasso underground laboratory.

Acknowledgements. We would like to warmly thank the many technical collaborators that contributed to the construction of the detector and to its operation. We are glad of the financial and technical support of our funding agencies and in particular of the Istituto Nazionale di Fisica Nucleare (INFN), of ETH Zürich and of the Fonds National Suisse de la Recherche Scientifique, Switzerland. This work has been supported by the CICYT Grant FPA2002-01835. The Polish groups acknowledge the support of the State Committee for Scientific Research in Poland, 105,160,620,621/E-344,E-340, E-77,E78/SPS/ICARUS/P-03/DZ211-214/2003-2005 and 1P03B 041 30; the INFN, FAI program; the EU Commission, TA-DUSLP2004-08-LNGS.

\section{References}

1. ICARUS Collaboration, S. Amerio et al., Nucl. Instrum. Methods A 527, 329 (2004)

2. ICARUS Collaboration, S. Amoruso et al., Nucl. Instrum. Methods A 523, 275 (2004) 
3. ICARUS Collaboration, S. Amoruso et al., Nucl. Instrum. Methods A 516, 68 (2004)

4. ICARUS Collaboration, M. Antonello et al., Nucl. Instrum. Methods A 516, 348 (2004)

5. ICARUS Collaboration, S. Amoruso et al., Eur. Phys. J. C 33, 233 (2004)

6. ICARUS Collaboration, F. Arneodo et al., Nucl. Instrum. Methods A 508, 287 (2003)

7. ICARUS Collaboration, F. Arneodo et al., Nucl. Instrum. Methods A 516, 610 (2004)

8. ICARUS Collaboration, F. Arneodo et al., LNGS P28/01, LNGS-EXP 13/89 add. 1/01, [arXiv:hep-ex/0103008]
9. R.E. Kalman, Trans. ASME D J. Basic Eng. 82, 35 (1960)

10. ICARUS Collaboration, P. Cennini et al., Nucl. Instrum. Methods A 432, 240 (1999)

11. G. Battistoni, A. Ferrari, T. Montaruli, P. Sala, Astropart. Phys. 19, 269 (2003), see http://www.fluka.org/ for further details

12. Particle Data Group, S. Eidelman et al., Phys. Lett. B 592, 1 (2004)

13. R. Fruhwirth, Nucl. Instrum. Methods A 262, 444 (1987)

14. E.J. Wolin, L.L. Ho, Nucl. Instrum. Methods A 329, 493 (1993) 